\title{
MHD Flow of Thermally Radiative Maxwell Fluid Past a Heated Stretching Sheet with Cattaneo-Christov Dual Diffusion
}

\author{
K. Loganathan $\mathbb{D}^{1},{ }^{1}$ Nazek Alessa $\mathbb{D}^{2},{ }^{2}$ Ngawang Namgyel $\mathbb{D}^{\circ},{ }^{3}$ and T. S. Karthik ${ }^{4}$ \\ ${ }^{1}$ Research and Development Wing, Live4Research, Tiruppur-638106, Tamilnadu, India \\ ${ }^{2}$ Department of Mathematical Sciences, Faculty of Science, Princess Nourah Bint Abdulrahman University, Riyadh, Saudi Arabia \\ ${ }^{3}$ Department of Humanities and Management, Jigme Namgyel Engineering College, Royal University of Bhutan, \\ Dewathang, Bhutan \\ ${ }^{4}$ Department of Electronics and Communication Engineering, Aditya College of Engineering and Technology, \\ Surampalem 533 437, Andhra Pradesh, India
}

Correspondence should be addressed to K. Loganathan; loganathankaruppusamy304@gmail.com and Ngawang Namgyel; ngawangnamgyel@jnec.edu.bt

Received 3 March 2021; Revised 5 June 2021; Accepted 9 June 2021; Published 23 June 2021

Academic Editor: Riaz Ahmad

Copyright $(\subset) 2021 \mathrm{~K}$. Loganathan et al. This is an open access article distributed under the Creative Commons Attribution License, which permits unrestricted use, distribution, and reproduction in any medium, provided the original work is properly cited.

\begin{abstract}
This study explains the impression of MHD Maxwell fluid with the presence of thermal radiation on a heated surface. The heat and mass transmission analysis is carried out with the available of Cattaneo-Christov dual diffusion. The derived PDE equations are renovated into ODE equations with the use of similarity variables. HAM technique is implemented for finding the solution. The importance of physical parameters of fluid velocity, temperature, concentration, skin friction, and heat and mass transfer rates are illustrated in graphs. We found that the fluid velocity declines with the presence of the magnetic field parameter. On the contrary, the liquid temperature enhances by increasing the radiation parameter. In addition, the fluid velocity is low, and temperature and concentration are high in Maxwell fluid compared to the viscous liquid.
\end{abstract}

\section{Introduction}

Many industrial processes depend on fluids especially, nonNewtonian fluids. Few examples are plastic sheet extrusion, paper production, spinning of metals, glass fiber, etc. Maxwell is one of the non-Newtonian models, and he predicts the stress relaxation. The primary principle of MHD is that forces are produced in the fluid when the magnetic field induces a current through a moving conducting fluid. Magnetohydrodynamics has diverse engineering applications. Sandeep et al. [1] examined the stretching surface flows of Oldroyd-B, Jeffrey, and Maxwell fluids with nonuniform heat source/sink impacts along with radiation effects. They found that Oldroyd-B and Maxwell fluids have lesser effects compared to the Jeffrey fluid. Farooq et al. [2] analyzed the exponentially stretching sheet flow of a Maxwell-type nanomaterial. The Buongiorno model was used in this study to construct the physical model. Fetecau et al. [3] discussed the porous channel flow of the upper-convected Maxwell (UCM). Also, steady-state transient components have an appearance of oscillatory motion. Wang et al. [4] and Sun et al. [5] established the incompressible Maxwell fluid passed through a tube through a triangular cross section (rectangular or isosceles). Analytical approaches are implemented for steady-state solutions of two oscillatory motions. Few other studies about the Maxwell fluid types have been implemented by Qi and Xu [6], Wenchang et al. [7], and Qi and Liu [8].

Heat transfer is a natural phenomenon of heat owing between the object or within the object in the order of the temperature difference. This phenomenon has a wide application in enormous fields such as semiconductors, cooling devices, and power generation. In earlier days, heat transfer is characterized by the Fourier law of heat conduction [9]. However, this law fails to explain the heat transfer effect, and in nature, no material will satisfy this law. So, Catteneo [10] 
extended the work of Fourier by including the thermal relaxation time. Later, Christov [11] upgraded Catteneo's work with the help of Oldroyd's upper-convected derivatives and thermal relaxation time for efficient performance. Saleem et al. [12] investigated the 3D combined convective Maxwell fluid with mass and heat Catteneo-Christov heat flux models with heat generation. Loganathan et al. [13] presented the second-order slip phenomenon of Oldroyd-B fluid with cross diffusion, radiation, and Catteneo-Christov heat flux impacts. Magento-free convection of nanoliquid flow towards a vertical cone, vertical wedge, and a vertical plate with Catteneo-Christov heat flux was studied by Jayachandra et al. [14]. Some other cutting edge research reports in this area can be found in [15-22].

The emission of electromagnetic waves from a material with a temperature greater than zero is known as thermal radiation. Solar radiation from the Sun and the infrared radiation emitted by the household products are examples of thermal radiation. The study of thermally radiative flow over a stretchy plate plays a vital role in many engineering applications, such as disposals of nuclear waste, drying the food products, film cooling, radial diffusers, gas turbines, and power plants. Ali et al. [23] investigated the thermal radiation properties of Newtonian-type nanofluid with stagnation point, combined convection, Joule heating, and stratification. Pal and Mandal [24] evaluated the porous medium flow of radiative nanoliquid with viscous dissipation and combined convection. They find double solutions for the shrinking case. Haroun et al. [25] presented the new process named spectral relaxation method (SRM) to solute the problem of MHD nanofluid flow with mixed convection. The fluid flow with thermal radiation over different geometries and situations is reported [26-30].

The authors examine the impression of CattaneoChristov dual diffusion of a non-Newtonian Maxwell fluid with the magnetic field, heat generation, and thermal radiation past a heated surface. The homotopy analysis method (HAM) [31-36] is employed to solute the physical system. Visualization of physical parameters reported and discussed in detail.

\section{Formulation of Physical Problem}

We analyze the 2D flow of a MHD Maxwell fluid over an extended sheet. Energy and mass determinations are calculated with Cattaneo-Christov dual diffusion. Let $T_{\infty}$ and $C_{\infty}$ be the free stream fluid temperature and fluid concentration which is lower than the fluid temperature and fluid concentration $T_{w}$ and $C_{w}$, respectively. The lowermost part of the plate was heated with hot fluid $T_{f}$. Figure 1 shows the physical representation of the flow problem. The governing mathematical models with the above assumptions are

$$
\begin{aligned}
\frac{\partial u_{1}}{\partial x}+\frac{\partial v_{1}}{\partial y}= & 0 \\
u_{1} \frac{\partial u_{1}}{\partial x}+v_{1} \frac{\partial u_{1}}{\partial y}= & \nu \frac{\partial^{2} u_{1}}{\partial y^{2}}-\beta\left(u_{1}^{2} \frac{\partial^{2} u_{1}}{\partial x^{2}}+v_{1}^{2} \frac{\partial^{2} u_{1}}{\partial y^{2}}+2 u_{1} v_{1} \frac{\partial^{2} u_{1}}{\partial x \partial y}\right)-\frac{\sigma B_{0}^{2}}{\rho} u_{1} \\
u_{1} \frac{\partial T}{\partial x}+v_{1} \frac{\partial T}{\partial y}= & \frac{k_{T}}{\rho c_{p}}\left[1+\frac{16 \sigma^{*} T_{\infty}^{3}}{3 \rho c_{p} k}\right] \frac{\partial^{2} T}{\partial y^{2}}-\lambda_{T}\left(u_{1}^{2} \frac{\partial^{2} T}{\partial x^{2}}+v_{1}^{2} \frac{\partial^{2} T}{\partial y^{2}}+\left(u_{1} \frac{\partial u_{1}}{\partial x}+v_{1} \frac{\partial u_{1}}{\partial y}\right) \frac{\partial T}{\partial x}+2 u_{1} v_{1} \frac{\partial T^{2}}{\partial x \partial y}\right) \\
& +\left(u_{1} \frac{\partial u_{1}}{\partial x}+v_{1} \frac{\partial u_{1}}{\partial y}\right) \frac{\partial T}{\partial y}+\frac{Q}{\rho c_{p}}\left(T-T_{\infty}\right) \\
u_{1} \frac{\partial C}{\partial x}+v_{1} \frac{\partial C}{\partial y}= & D_{B} \frac{\partial^{2} C}{\partial y^{2}}-\lambda_{c}\left(u_{1} \frac{\partial^{2} C}{\partial x^{2}}+v_{1}^{2} \frac{\partial^{2} C}{\partial y^{2}}+\left(u_{1} \frac{\partial u_{1}}{\partial x}+v_{1} \frac{\partial u_{1}}{\partial y}\right) \frac{\partial C}{\partial x}+2 u_{1} v_{1} \frac{\partial C^{2}}{\partial x \partial y}\right)+\left(u_{1} \frac{\partial u_{1}}{\partial x}+v_{1} \frac{\partial u_{1}}{\partial y}\right) \frac{\partial C}{\partial y} \\
u_{1}= & u_{1_{w}}, v_{1}=-v_{1_{w}},-k_{T} \frac{\partial T}{\partial y}=h_{f}\left(T_{f}-T_{\infty}\right), C=C_{w} \text { at } y=0 \\
u_{1} \longrightarrow & 0, C \longrightarrow C_{\infty}, T \longrightarrow T_{\infty} \text { as } y \longrightarrow \infty
\end{aligned}
$$

Consider the following similarity transformations: 


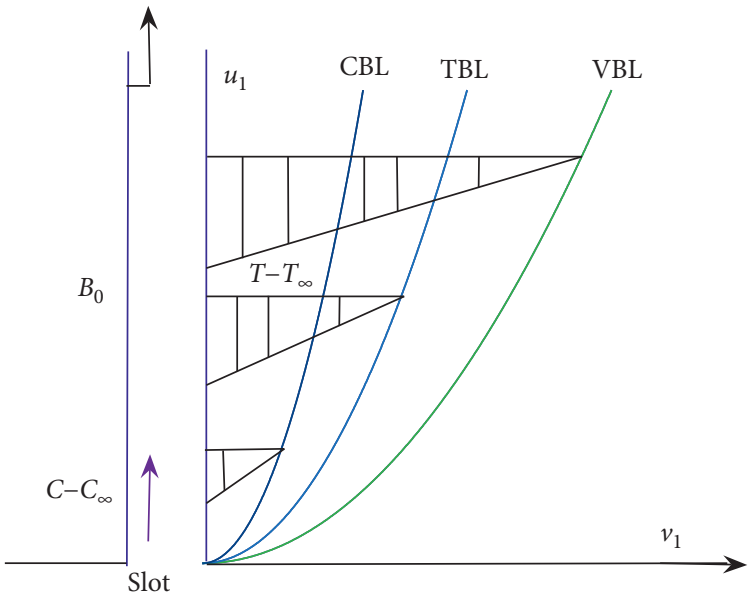

Figure 1: Schematic representation of Maxwell fluid flow.

$$
\begin{aligned}
\psi & =\sqrt{a v} x f(\eta), u_{1}=\frac{\partial \psi}{\partial y}, v_{1}=-\frac{\partial \psi}{\partial x}, \eta=\sqrt{\frac{a}{v}} y, \\
u_{1} & =a x f^{\prime}(\eta), v_{1}=-\sqrt{a v} f(\eta),
\end{aligned}
$$

$\theta(\eta)=\frac{T-T_{\infty}}{T_{f}-T_{\infty}}, \phi(\eta)=\frac{C-C_{\infty}}{C_{w}-C_{\infty}}$.

Apply the above transformations:

$$
f^{\prime \prime \prime}+f f^{\prime \prime}-f^{2}+\lambda\left(2 f f^{\prime} f^{\prime \prime}-f^{2} f^{\prime \prime \prime}\right)-M f^{\prime}=0,
$$

$$
\begin{array}{r}
\left(1+\frac{4}{3} R\right) \theta^{\prime \prime}+\operatorname{Pr} f \theta^{\prime}-\operatorname{Pr} \gamma\left(f^{2} \theta^{\prime \prime}+f f^{\prime} \theta^{\prime}\right)+\operatorname{Pr} H g \theta=0 \\
\frac{1}{S c} \phi^{\prime \prime}+f \phi^{\prime}-\gamma_{c}\left(f^{2} \phi^{\prime \prime}+f f^{\prime} \phi^{\prime}\right)=0
\end{array}
$$

$f(\eta)=f w, f^{\prime}(\eta)=1, \theta^{\prime}(\eta)=-B i(1-\theta(0))$,

$\phi(\eta)=1$ at $y=0$,

$f^{\prime}(\eta) \longrightarrow 0, \theta(\eta) \longrightarrow 0, \phi(\eta) \longrightarrow 0$ as $y \longrightarrow \infty$.

The nondimensional variables are declared as

$$
\begin{aligned}
& \lambda=\beta a, \operatorname{Pr}=\frac{\nu \rho C_{p}}{k_{T}}, R d=\frac{\left(4 \sigma^{*} T_{\infty}^{3}\right)}{\left(k k_{T}\right)}, M=\frac{\sigma B_{0}^{2}}{\rho a}, H g=\frac{Q}{a \rho c_{p}}, \\
& \gamma=\lambda_{T} a, \gamma_{c}=\lambda_{c} a, B i=\frac{h_{f}}{k} \sqrt{\frac{v}{a}}, S c=\frac{\nu}{D_{B}} .
\end{aligned}
$$

The engineering quantities are stated as

$$
\begin{aligned}
\frac{1}{2} C_{f} \sqrt{\mathrm{Re}} & =(1+\lambda) f^{\prime \prime}(0) \text { ("Local skin friction”), } \\
\frac{N u}{\sqrt{\mathrm{Re}}} & =-\left(1+\frac{4}{3} R\right) \theta^{\prime}(0) \text { ("Local Nusselt number”), } \\
\frac{S h}{\sqrt{\mathrm{Re}}} & =-\phi^{\prime}(0) \text { ("Local Sherwood number"). }
\end{aligned}
$$

\section{HAM Solution}

Several numerical schemes are available for solute the nonlinearity problems. The efficient semianalytic process HAM was employed to solute these current nonlinearity problems. This method presents the independence to select the primary assumptions of the solutions.

The initial guesses are

$$
\begin{aligned}
& f_{0}=f w+1-e^{-\eta}, \\
& \theta_{0}=\frac{B i e^{-\eta}}{1+B i} \\
& \phi_{0}=e^{-\eta} .
\end{aligned}
$$

Linear operators are

$$
\begin{aligned}
L_{f} & =f^{\prime \prime \prime}(\eta)-f^{\prime}(\eta), L_{\theta}=\theta^{\prime \prime}(\eta)-\theta(\eta) \text { and } L_{\phi}=\phi^{\prime \prime}(\eta)-\phi(\eta), \\
L_{f}\left[\Delta_{1}+\Delta_{2} e^{\eta}+\Delta_{3} e^{-\eta}\right] & =0, L_{\theta}\left[\Delta_{4} e^{\eta}+\Delta_{5} e^{-\eta}\right]=0, \text { and } L_{\phi}\left[\Delta_{6} e^{\eta}+\Delta_{7} e^{-\eta}\right]=0,
\end{aligned}
$$

where $\Delta_{j}(j=1-7)$. 


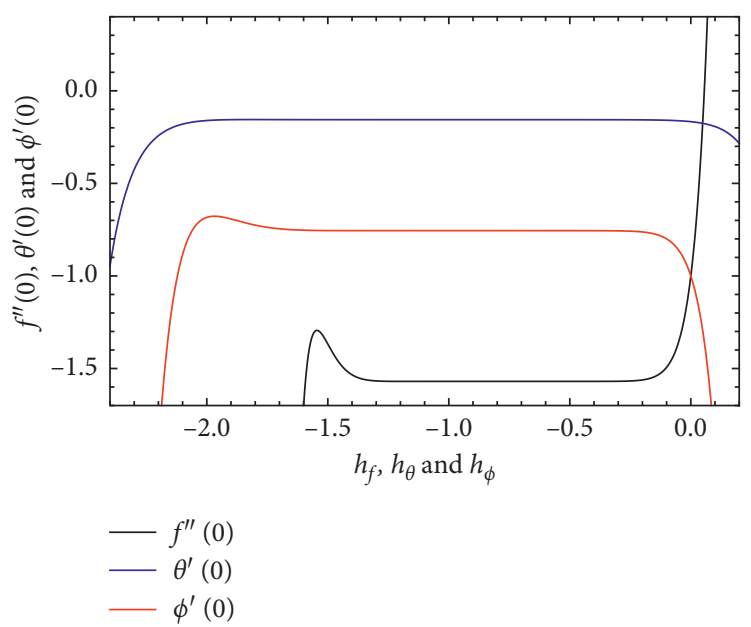

Figure 2: h-curves for $\mathbf{h}_{f}, \mathbf{h}_{\theta}$, and $\mathbf{h}_{\phi}$.

TABLE 1: Order of approximations of HAM.

\begin{tabular}{lllr}
\hline Order & $-f^{\prime \prime}(0)$ & $-\theta^{\prime}(0)$ & $-\phi^{\prime}(0)$ \\
\hline 1 & 1.43040 & 0.16076 & 0.82567 \\
5 & 1.56923 & 0.15667 & 0.76175 \\
10 & 1.56956 & 0.15605 & 0.75586 \\
15 & 1.56956 & 0.15596 & 0.75532 \\
20 & 1.56956 & 0.15596 & 0.75532 \\
25 & 1.56956 & 0.15596 & 0.75532 \\
30 & 1.56956 & 0.15596 & 0.75532 \\
35 & 1.56956 & 0.15596 & 0.75532 \\
40 & 1.56956 & 0.15596 & 0.75532 \\
\hline
\end{tabular}

Generally, the HAM solution depends on the auxiliary parameters $h_{f}, h_{\theta}$, and $h_{\phi}$, and these parameters control the convergence. The range value of $h_{f}, h_{\theta}$, and $h_{\phi}$ are $-1.4 \leq h_{f} \leq-0.2,-0.2 \leq h_{\theta} \leq 0$, and $-1.8 \leq h_{\phi} \leq-0.2$. We fix $h_{f}=-0.8$ and $h_{\theta}=h_{\phi}=-1$ for better convergence (see Figure 2). Table 1 provides the order of HAM, and we found that $15^{\text {th }}$ order is adequate for all profiles. Table 2 indicates the code validation of $f^{\prime \prime}(0)$ for various values of $\lambda$ with the limiting value of $M=0$.

\section{Result and Discussion}

In this section, we focus on the importance of physical parameters of fluid velocity, fluid temperature, fluid concentration, skin friction coefficient, local Nusselt number, and local Sherwood number for viscous fluid $(\lambda=0.0)$ and Maxwell fluid $(\lambda=0.4)$. Figures 3(a)-3(d) show the impact of $\lambda, M, f w(+)$, and $f w(-)$ on velocity profile for both viscous and Maxwell fluids. Figure 3(a) provides the impact of Deborah number $(\lambda)$ on the velocity of the fluid. The fluid velocity improves while boosting up the values of $\lambda$. For Newtonian fluids, the opposite effect is produced by $\lambda$, for these type of fluids, the boundary layer thickness rises for higher $\lambda$. Figure 3 elucidates the magnetic field $(M)$ influence on velocity of the fluid. The higher range of $M$ produces a reduction in velocity of the fluid because the Lorentz force is created against the fluid flow by magnetic field. The maximum Lorentz force is produced while the magnetic field is applied at perpendicular to the fluid flow. For this reason, momentum boundary layer thickness reduces. The fluid velocity suppresses with more availability of suction, and it enhances in the injection case (see Figures 3(c) and $3(\mathrm{~d}))$.

The significance of " $\gamma, R, B i$, and $f w$ " on fluid temperature profile for both fluids was illustrated in Figures 4(a)-4(f) and seen that the fluid temperature becomes high with enhancing the values of " $R, B i>=0.0$ and $f w<=0.0$ " for both fluids. However, it decreases for " $f w>=0.0$ and $B i<=0.0$." In addition, the thickness of the thermal boundary layer is high in Maxwell fluid compared to viscous fluid. Figures 5(a)-5(d) provide the variance of fluid 
TABLE 2: Code validation results with $f^{\prime \prime}(0)$ obtained by Mukhopadhyay [37] and Sadghey et al. [38] in the limiting condition for various $\lambda$ by fixing $M=0$.

\begin{tabular}{lccc}
\hline$\lambda$ & Mukhopadhyay [37] & Sadghey et al. [38] & Present \\
\hline 0.0 & 0.9999963 & 1.000 & 1.00000 \\
0.2 & 1.051949 & 1.0549 & 1.05189 \\
0.4 & 1.101851 & 1.10084 & 1.10190 \\
0.6 & 1.150162 & 1.0015016 & 1.15014 \\
0.8 & 1.196693 & 1.19872 & 1.19671 \\
\hline
\end{tabular}

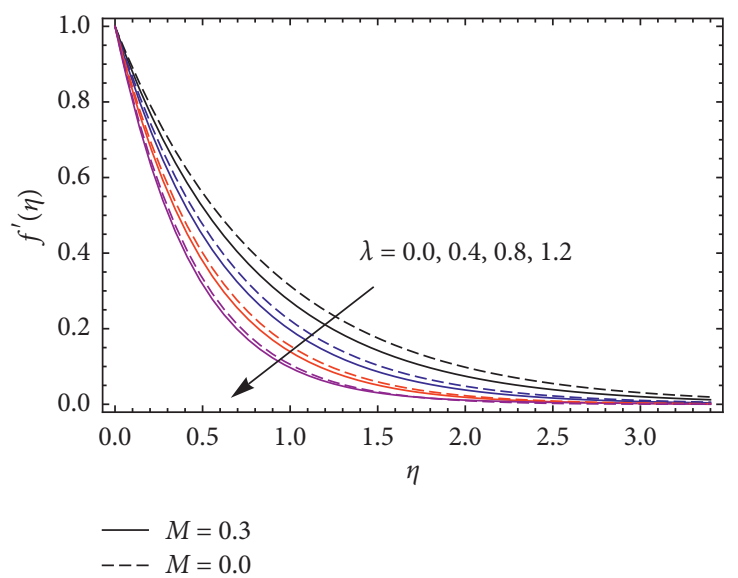

(a)

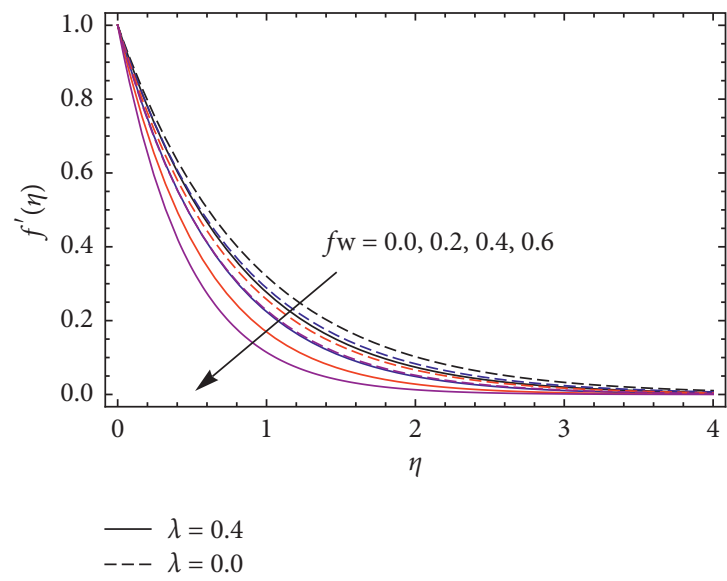

(c)

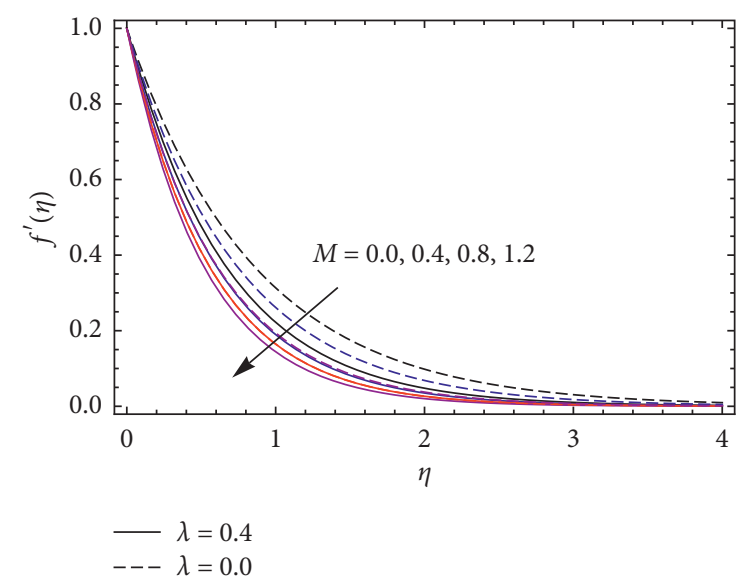

(b)

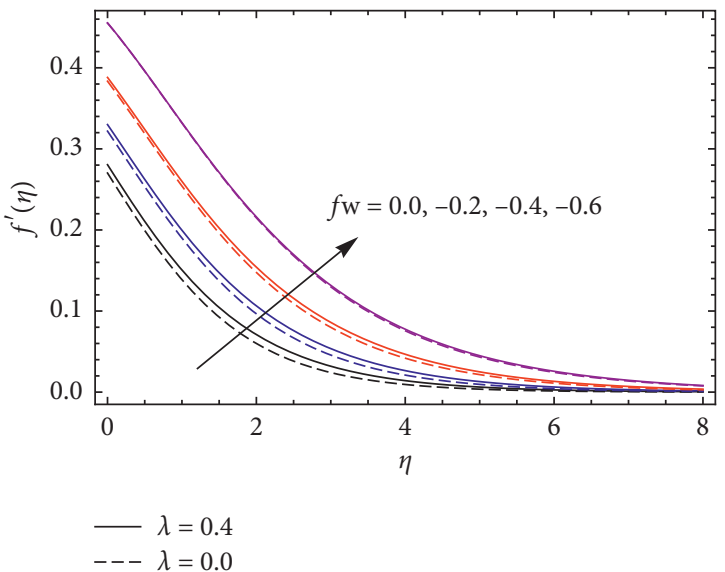

(d)

Figure 3: Impact of velocity on the different values of parameters. (a) $\lambda=0.0,0.4,0.8,1.2$, (b) $M=0.0,0.4,0.8,1.2,(\mathrm{c})$ $f w=0.0,0.2,0.4,0.6$, and $(\mathrm{d}) f w=-0.0,-0.2,-0.4,-0.6$.

concentration for " $\gamma_{c}, f w$, and $S c$ " for both fluids. It is noted that the fluid concentration is a nondecreasing function of injection, and the reverse trends were obtained in " $\gamma_{c}>=0, f w$, and $S c$ " for both fluids. It is also noted that the concentration boundary layer is high in Maxwell fluid compared to viscous fluid.

The skin friction coefficient for different combination of " $f w, M$, and $\lambda$ " was shown in Figures 6(a) and 6(b). It is concluded that the surface shear stress declines with increasing values of " $f w, M$, and $\lambda$ ". Figures 7 (a) and $7(\mathrm{~b})$ explain the local Nusselt number for different combinations of " $B i$, lambda, $R$, and $\gamma$." It is found that the heat transfer gradient raises with escalating the values of " $B i, \gamma$, and $R$," and it decreases with heightening the values of " $\lambda$." The local Sherwood number for different combinations of " $\gamma_{c}, M, \lambda$, and Sc" are presented in Figures 8(a) and 8(b). We noted that the mass transfer gradient becomes small with rising the values of " $M$ and $\lambda$ " and it is high for the presence of " $\gamma_{c}$ and Sc." 

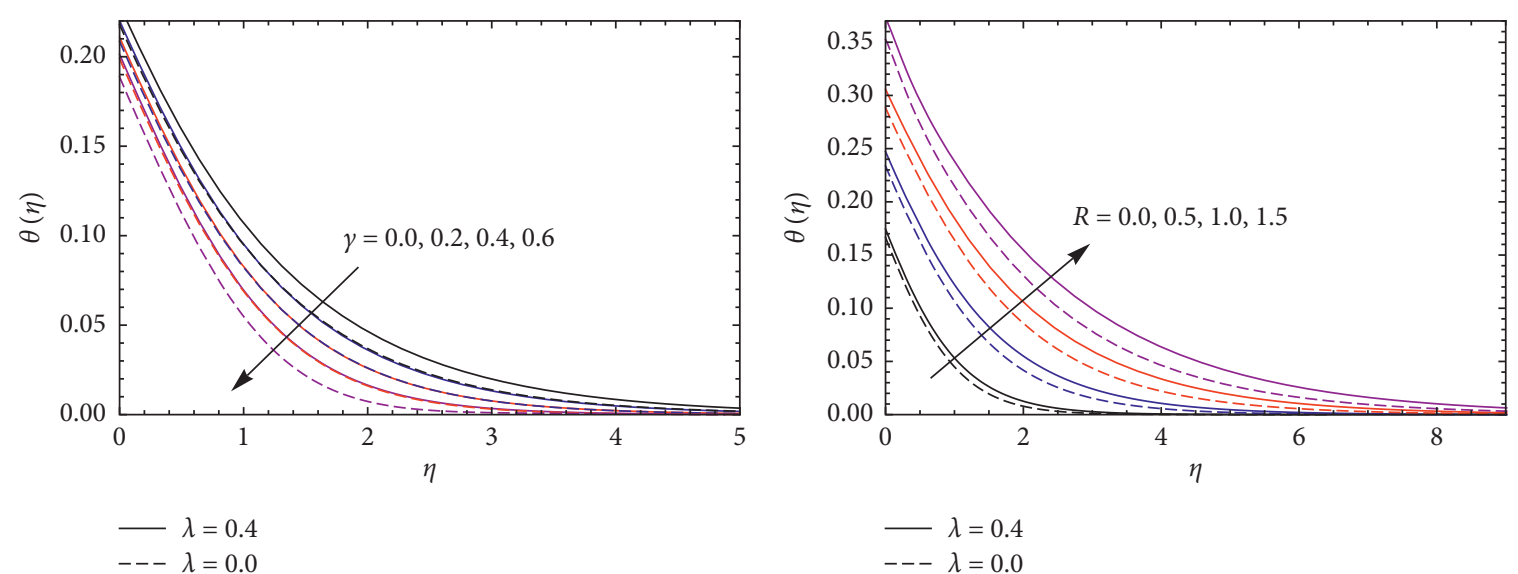

(a)
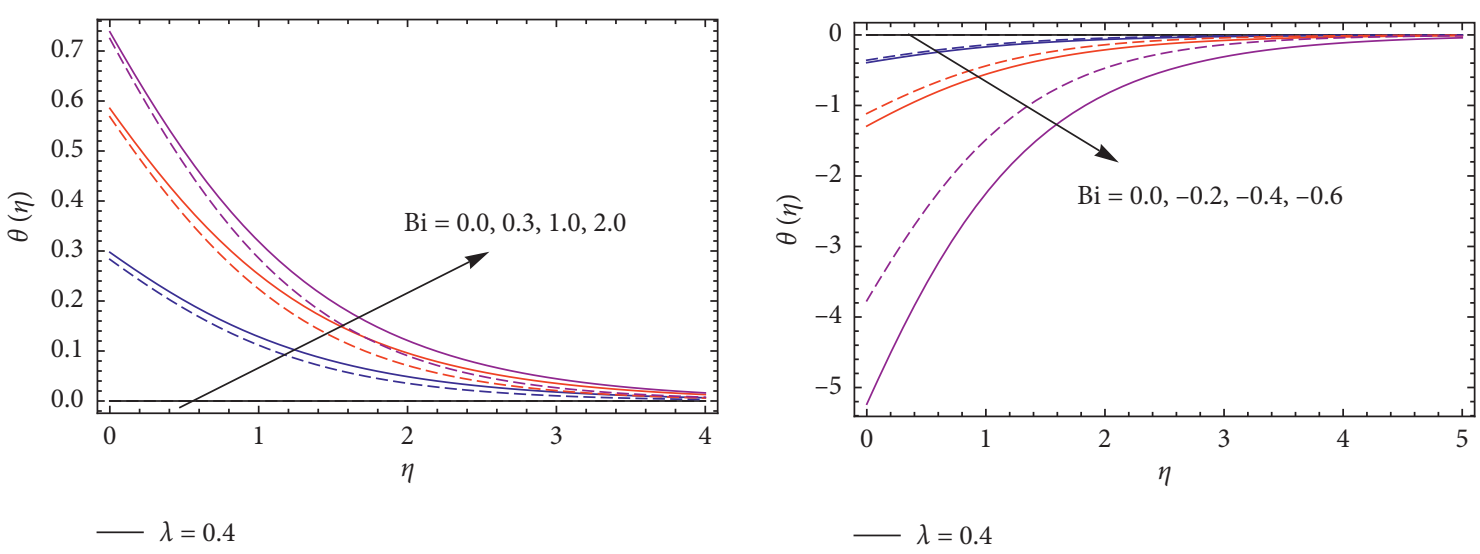

$\lambda=0.4$

$--\lambda=0.0$

$--\lambda=0.0$

(c)

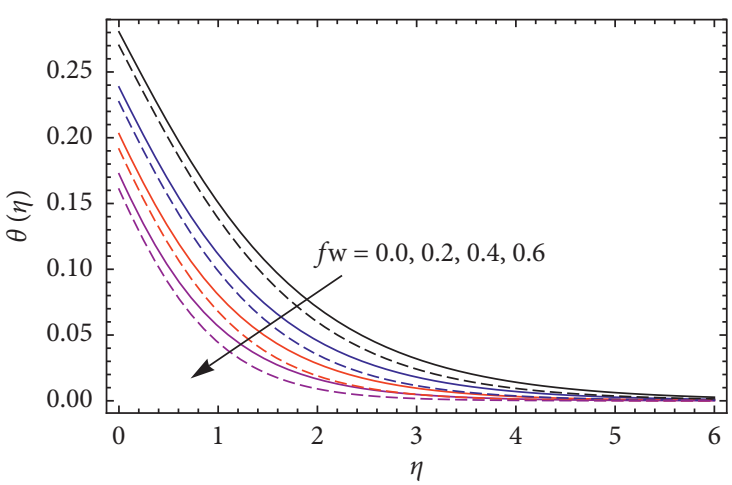

(d)

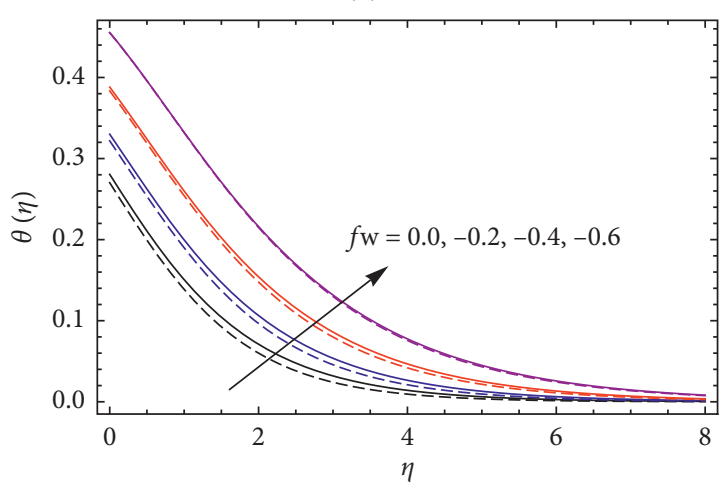

$-\lambda=0.4$

$-\lambda=0.4$

--- $\lambda=0.0$

--- $\lambda=0.0$

(e)

(f)

FIgURE 4: Impact of temperature on the different values of parameters. (a) $\gamma=0.0,0.2,0.4,0.6, \quad$ (b) $R=0.0,0.5,1.0,1.5$, (c) $B i=0.0,0.3,1.0,2.0$, (d) $B i=0.0,-0.2,-0.4,-0.6$, (e) $f w=0.0,0.2,0.4,0.6$, and (f) $f w=0.0,-0.2,-0.4,-0.6$. 

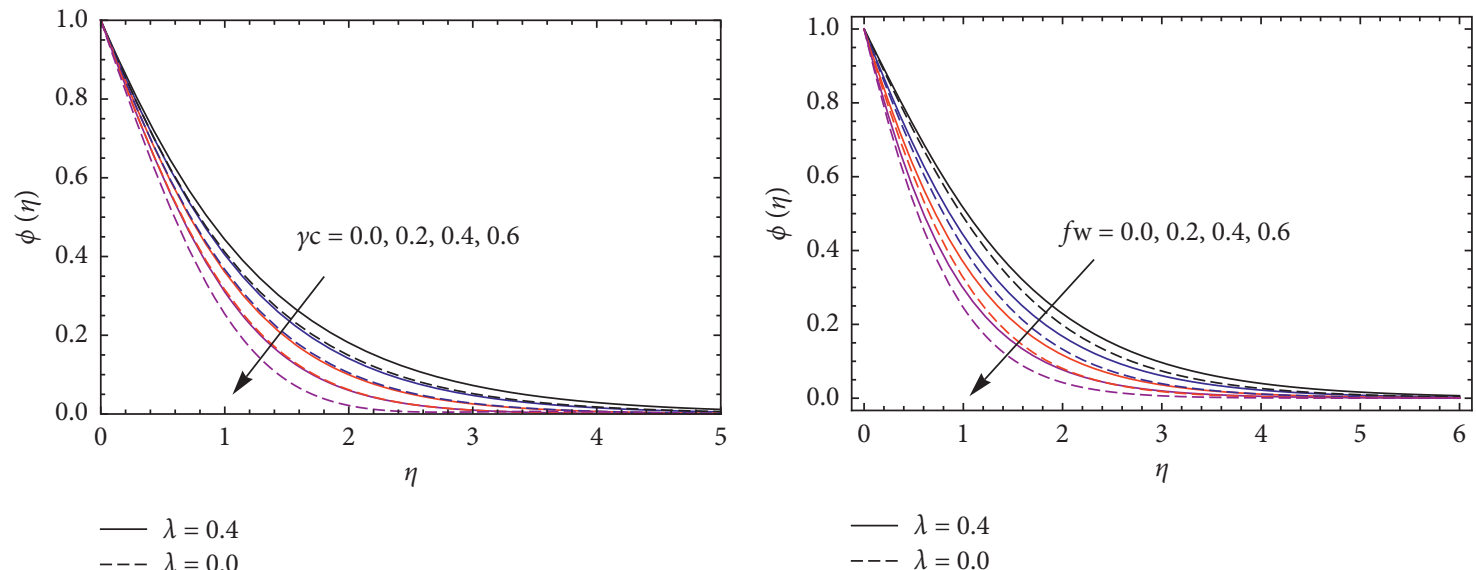

(a)
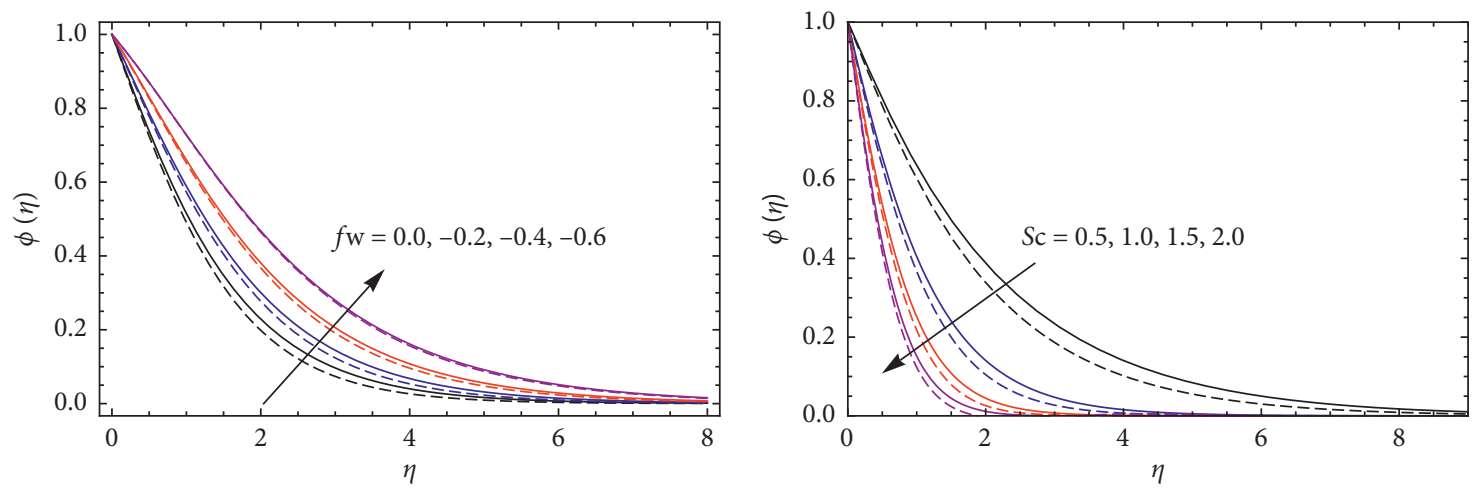

$\begin{aligned}-\lambda & =0.4 \\ --\lambda & =0.0\end{aligned}$

$\begin{aligned}-\lambda & =0.4 \\ --\lambda & =0.0\end{aligned}$

(c)

(d)

FiguRE 5: Impact of concentration on the different values of parameters. (a) $\gamma_{c}=0.0,0.2,0.4,0.6$, (b) $f w=0.0,0.2,0.4,0.6$, (c) $f w=0.0,-0.2,-0.4,-0.6$, and (d) $S c=0.5,1.0,1.5,2.0$.

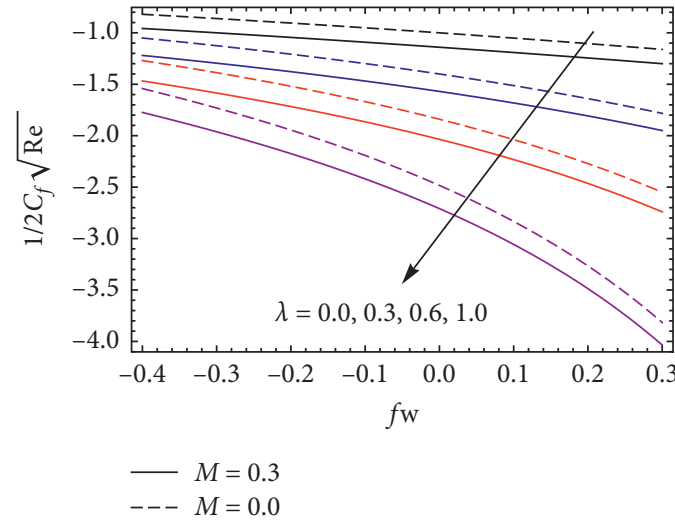

(a)

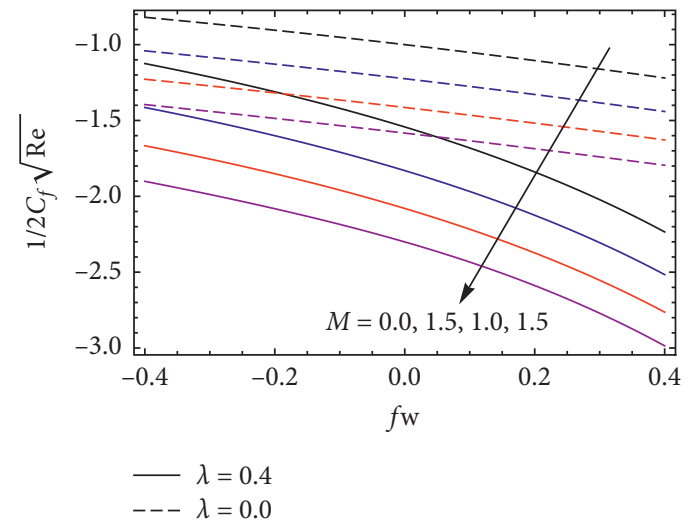

(b)

Figure 6: Impact of skin friction on combined parameters. (a) $\lambda$ and $f w$ and (b) $M$ and $f w$. 


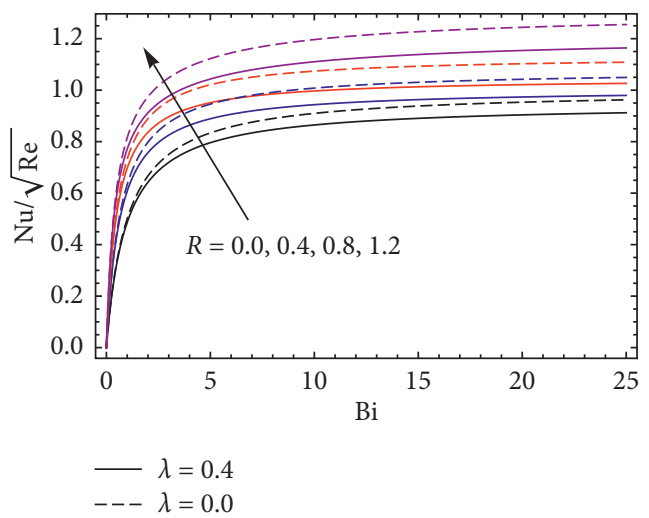

(a)

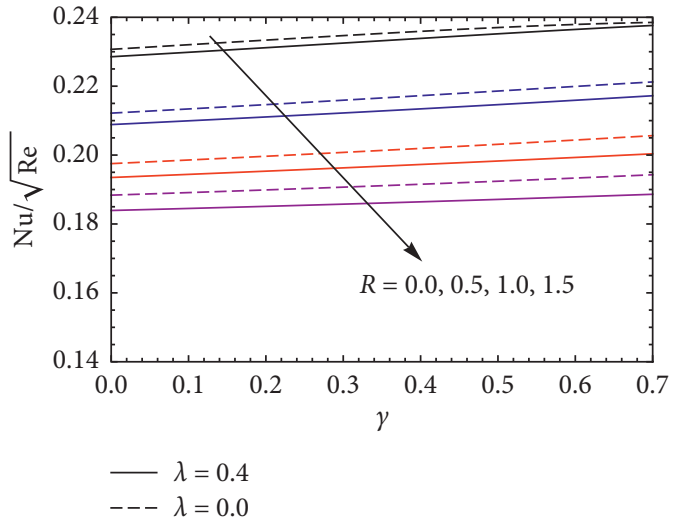

(b)

Figure 7: Impact of the Nusselt number on combined parameters. (a) $R$ and $B i$ and (b) $R$ and $\gamma$.

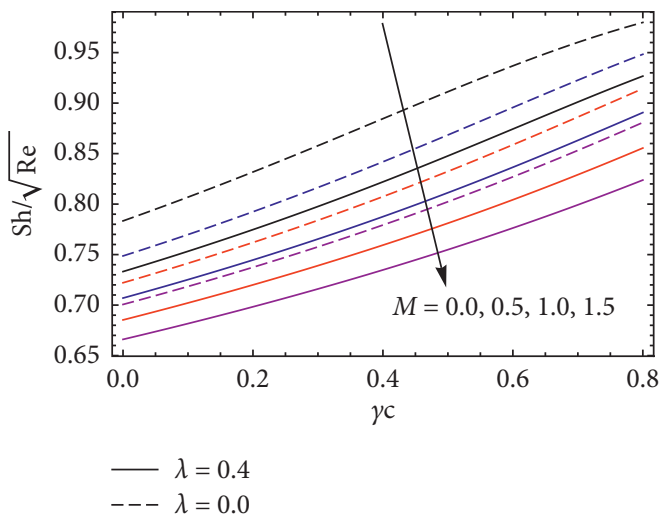

(a)

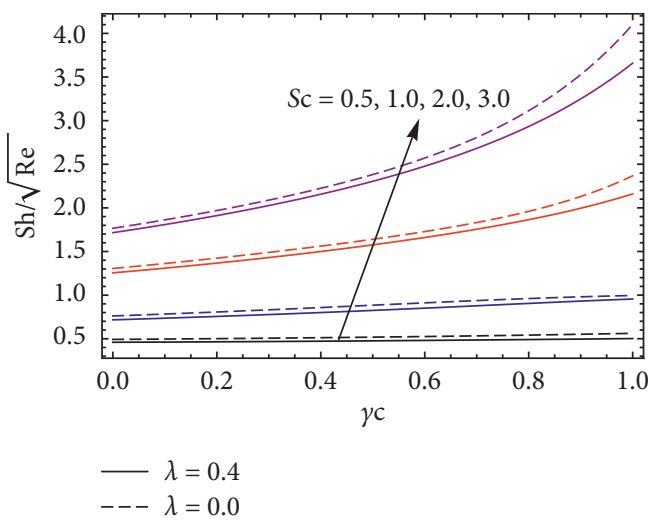

(b)

Figure 8: Impact of the Sherwood number on combined parameters. (a) $\gamma_{c}$ and $M$ and (b) $\gamma_{c}$ and Sc.

\section{Conclusions}

In our analysis, we found the following key points:

(i) The fluid velocity declines with escalating the value of magnetic field parameter $(M)$ and Maxwell fluid parameter $(\lambda)$

(ii) Rising values of thermal relaxation time and Biot number leads to the higher heat transfer rate

(iii) The fluid concentration suppresses by enhancing the values solutal relaxation time

(iv) The surface shear stress is suppressing function of $M$ and $f w$

(v) The mass transfer gradient enhances with improving the solutal relaxation time parameter
Abbreviations
$a: \quad$ Stretching rate $\left(s^{-1}\right)$
$B i$ : Biot number
$B_{0}: \quad$ Constant magnetic field $\left(\mathrm{kgs}={ }^{2} A^{-1}\right)$
C: $\quad$ Concentration $\left(\mathrm{kgm}^{-3}\right)$

$c_{p}: \quad$ Specific heat $\left(\mathrm{J} \mathrm{kg}^{-1} \mathrm{~K}^{-1}\right)$

$C_{\infty}:$ Ambient concentration $\left(\mathrm{kgm}^{-3}\right)$

$C_{w}: \quad$ Fluid wall concentration $\left(\mathrm{kgm}^{-3}\right)$

$C_{f}: \quad$ Skin friction coefficient

$D_{B}$ : $\quad$ Mass diffusivity $\left(\mathrm{m}^{2} \mathrm{~s}^{-1}\right)$

$f(\eta)$ : Velocity similarity function

$f w$ : Suction / injection parameter

$\mathrm{Hg}$ : Heat generation parameter

$h_{f}: \quad$ Convective heat transfer coefficient $\left(W^{-1} \mathrm{~K}^{-1}\right)$

$k_{T}$ : Thermal conductivity $\left(W m^{-1} K^{-1}\right)$

$M$ : $\quad$ Hartmann number

$\mathrm{Nu}$ : Nusselt number

Pr: Prandtl number

Q: $\quad$ Dimensional heat generation/absorption coefficient

$R: \quad$ Radiation parameter

Sc: $\quad$ Schmidt number

Sh: $\quad$ Sherwood number

T: $\quad$ Temperature $(K)$

$T_{\infty}: \quad$ Ambient temperature $(K)$

$T_{f}$ : Convective surface temperature $(K)$

$u_{1_{w}}: \quad$ Velocity of the sheet $\left(\mathrm{ms}^{-1}\right)$

$u_{1}, v_{1}$ : Velocity components in $(x, y)$ directions $\left(\mathrm{ms}^{-1}\right)$ 
$v_{1}>0$ : Suction velocity

$v_{1_{w}}<0$ : Injection velocity

$x, y: \quad$ Cartesian coordinates $(m)$

$\beta: \quad$ Relaxation time of the fluid

$\phi(\eta)$ : Concentration similarity function

$\gamma: \quad$ Dimensionless thermal relaxation time

$\gamma_{c}: \quad$ Dimensionless mass relaxation time

$\eta: \quad$ Similarity parameter

$\lambda_{T}: \quad$ Thermal relaxation time

$\lambda_{c}: \quad$ Mass relaxation time

$\lambda: \quad$ Maxwell fluid parameter

$\nu: \quad$ Kinematic viscosity $\left(\mathrm{m}^{2} \mathrm{~s}^{-1}\right)$

$\theta(\eta)$ : Temperature similarity function

$\rho: \quad$ Density $\left(\mathrm{kgm}^{-1}\right)$

$\sigma: \quad$ Electrical conductivity $(\mathrm{Sm})$

$\psi$ : $\quad$ Stream function $\left(\mathrm{ms}^{-1}\right)$.

\section{Data Availability}

The raw data supporting the conclusions of this article will be made available by the authors without undue reservation.

\section{Conflicts of Interest}

The authors declare that they have no conflicts of interest.

\section{Acknowledgments}

This research was funded by the Deanship of Scientific Research at Princess Nourah Bint Abdulrahman University through the Fast-track Research Funding Program.

\section{References}

[1] N. Sandeep and C. Sulochana, "Momentum and heat transfer behaviour of Jeffrey, Maxwell and Oldroyd-B nanofluids past a stretching surface with non-uniform heat source/sink," Ain Shams Engineering Journal, vol. 9, no. 4, pp. 517-524, 2018.

[2] U. Farooq, D. Lu, S. Munir, M Ramzan, M Suleman, and S Hussain, "MHD flow of Maxwell fluid with nanomaterials due to an exponentially stretching surface," Scientific Reports, vol. 9, Article ID 7312, 2019.

[3] C. Fetecau, R. Ellahi, and S. M. Sait, "Mathematical analysis of Maxwell fluid flow through a porous plate channel induced by a constantly accelerating or oscillating wall," Mathematics, vol. 9, no. 1, p. 90, 2021.

[4] S. Wang, P. Li, and M. Zhao, "Analytical study of oscillatory flow of Maxwell fluid through a rectangular tube," Physics of Fluids, vol. 31, Article ID 063102, 2019.

[5] X. Sun, S. Wang, and M. Zhao, "Oscillatory flow of Maxwell fluid in a tube of isosceles right triangular cross section," Physics of Fluids, vol. 31, Article ID 123101, 2019.

[6] H. Qi and M. Xu, "Unsteady flow of viscoelastic fluid with fractional Maxwell model in a channel," Mechanics Research Communications, vol. 34, no. 2, pp. 210-212, 2007.

[7] T. Wenchang, P. Wenxiao, and X. Mingyu, "A note on unsteady flows of a viscoelastic fluid with the fractional Maxwell model between two parallel plates," International Journal of Non-linear Mechanics, vol. 38, no. 5, pp. 645-650, 2003.
[8] H. T. Qi and J. G. Liu, "Some duct flows of a fractional Maxwell fluid," The European Physical Journal Special Topics, vol. 193, no. 1, pp. 71-79, 2011.

[9] Chez Firmin Didot, Fourier, Theorie Analytique De La Chaleur, Chez Firmin Didot, Paris, France, 1822.

[10] C. Cattaneo, "Sulla Conduzionedelcalore," Atti del Seminario Matematico e Fisico dell Universita di Modena e Reggio Emilia, vol. 3, pp. 83-101, 1948.

[11] C. I. Christov, "On frame indifferent formulation of the Maxwell-Cattaneo model of finite-speed heat conduction," Mechanics Research Communications, vol. 36, no. 4, pp. 481-486, 2009.

[12] S. Saleem, M. Awais, S. Nadeem, N. Sandeep, and M. T. Mustafa, "Theoretical analysis of upper-convected Maxwell fluid flow with Cattaneo-Christov heat flux model," Chinese Journal of Physics, vol. 55, no. 4, pp. 1615-1625, 2017.

[13] K. Loganathan, S. Sivasankaran, M. Bhuvaneswari, and S. Rajan, "Second-order slip, cross-diffusion and chemical reaction effects on magneto-convection of Oldroyd-B liquid using Cattaneo-Christov heat flux with convective heating," Journal of Thermal Analysis and Calorimetry, vol. 136, no. 1, pp. 401-409, 2019.

[14] M. Jayachandra Babu, N. Sandeep, and S. Saleem, "Free convective MHD Cattaneo-Christov flow over three different geometries with thermophoresis and Brownian motion," Alexandria Engineering Journal, vol. 56, no. 4, pp. 659-669, 2017.

[15] K. Loganathan, K. Mohana, M. Mohanraj, P. Sakthivel, and S. Rajan, "Impact of third-grade nanofluid flow across a convective surface in the presence of inclined Lorentz force: an approach to entropy optimization," Journal of Thermal Analysis and Calorimetry, vol. 144, no. 5, pp. 1935-1947, 2020.

[16] T. Hayat, S. Qayyum, M. Imtiaz, and A. Alsaedi, "Impact of Cattaneo-Christov heat flux in Jeffrey fluid flow with homogeneous-heterogeneous reactions," PLOS ONE, vol. 11, Article ID e0148662, 2016.

[17] L. Liu, L. Zheng, F. Liu, and X. Zhang, "Anomalous convection diffusion and wave coupling transport of cells on comb frame with fractional Cattaneo-Christov flux," Communications in Nonlinear Science and Numerical Simulation, vol. 38, pp. 45-58, 2016.

[18] T. Salahuddin, M. Y. Malik, A. Hussain, S. Bilal, and M. Awais, "MHD flow of Cattanneo-Christov heat flux model for Williamson fluid over a stretching sheet with variable thickness: using numerical approach," Journal of Magnetism and Magnetic Materials, vol. 401, pp. 991-997, 2016.

[19] K. Loganathan and S. Rajan, "An entropy approach of Williamson nanofluid flow with Joule heating and zero nanoparticle mass flux," Journal of Thermal Analysis and Calorimetry, vol. 141, no. 6, pp. 2599-2612, 2020.

[20] S. Han, L. Zheng, C. Li, and X. Zhang, "Coupled flow and heat transfer in viscoelastic fluid with Cattaneo-Christov heat flux model," Applied Mathematics Letters, vol. 38, pp. 87-93, 2014.

[21] M. Mustafa, "Cattaneo-Christov heat flux model for rotating flow and heat transfer of upper-convected Maxwell fluid," AIP Advances, vol. 5, Article ID 047109, 2015.

[22] A. Hussain, M. Y. Malik, M. Khan, and T. Salahuddin, "Application of generalized Fourier heat conduction law on MHD viscoinelastic fluid flow over stretching surface," International Journal of Numerical Methods for Heat \& Fluid Flow, vol. 30, no. 6, pp. 3481-3496, 2020. 
[23] U. Ali, M. Y. Malik, A. A. Alderremy, S. Aly, and K. U. Rehman, "A generalized findings on thermal radiation and heat generation/absorption in nanofluid flow regime," Physica A: Statistical Mechanics and Its Applications, vol. 553, Article ID 124026, 2020.

[24] D. Pal and G. Mandal, "Influence of thermal radiation on mixed convection heat and mass transfer stagnation-point flow in nanofluids over stretching/shrinking sheet in a porous medium with chemical reaction," Nuclear Engineering and Design, vol. 273, pp. 644-652, 2014.

[25] N. A. Haroun, S. Mondal, and P. Sibanda, "Effects of thermal radiation on mixed convection in a MHD nanofluid flow over a stretching sheet using a spectral relaxation method," International Journal of Mathematical, Computational, Physical, Electrical and Computer Engineering, vol. 11, pp. 52-61, 2017.

[26] D. Pal and G. Mandal, "Double diffusive magnetohydrodynamic heat and mass transfer of nanofluids over a nonlinear stretching/shrinking sheet with viscous-Ohmic dissipation and thermal radiation," Propulsion and Power Research, vol. 6, no. 1, pp. 58-69, 2017.

[27] M. Khan, M. Irfan, and W. A. Khan, "Impact of nonlinear thermal radiation and gyrotactic microorganisms on the Magneto-Burgers nanofluid," International Journal of $\mathrm{Me}$ chanical Sciences, vol. 130, pp. 375-382, 2017.

[28] P. Sudarsana Reddy, A. J. Chamkha, and A. Al-Mudhaf, "MHD heat and mass transfer flow of a nanofluid over an inclined vertical porous plate with radiation and heat generation/absorption," Advanced Powder Technology, vol. 28, no. 3, pp. 1008-1017, 2017.

[29] M. Sheikholeslami and A. Ghasemi, "Solidification heat transfer of nanofluid in existence of thermal radiation by means of FEM," International Journal of Heat and Mass Transfer, vol. 123, pp. 418-431, 2018.

[30] K. Loganathan, N. Alessa, K. Tamilvanan, and F. S. Alshammari, "Significances of Darcy-Forchheimer porous medium in third-grade nanofluid flow with entropy features," The European Physical Journal Special Topics, 2021.

[31] S. J. Liao, Homotopy Analysis Method in Non-linear Differential Equations, Springer and Higher Education Press, Heidelberg, Germany, 2012.

[32] M. Turkyilmazoglu, "Solution of the Thomas-Fermi equation with a convergent approach," Communications in Nonlinear Science and Numerical Simulation, vol. 17, no. 11, pp. 40974103, 2012.

[33] U. Farooq, Y. L. Zhao, T. Hayat, A. Alsaedi, and S. J. Liao, "Application of the HAM-based Mathematica package BVPh 2.0 on MHD Falkner-Skan flow of nano-fluid," Computers \& Fluids, vol. 111, pp. 69-75, 2015.

[34] J. Sui, L. Zheng, X. Zhang, and G. Chen, "Mixed convection heat transfer in power law fluids over a moving conveyor along an inclined plate," International Journal of Heat and Mass Transfer, vol. 85, pp. 1023-1033, 2015.

[35] K. Loganathan, G. Muhiuddin, A. M. Alanazi, F. S. Alshammari, B. M. Alqurashi, and S. Rajan, "Entropy optimization of third-grade nanofluid slip flow embedded in a porous sheet with zero mass flux and a non-fourier heat flux model," Frontiers in Physics, vol. 8, p. 250, 2020.

[36] E. Elanchezhian, R. Nirmalkumar, M. Balamurugan, K. Mohana, K. M. Prabu, and A. Viloria, "Heat and mass transmission of an Oldroyd-B nanofluid flow through a stratified medium with swimming of motile gyrotactic microorganisms and nanoparticles," Journal of Thermal Analysis and Calorimetry, vol. 141, no. 6, pp. 2613-2623, 2020.
[37] S. Mukhopadhyay, "Heat transfer analysis of the unsteady flow of a Maxwell fluid over a stretching surface in the presence of a heat source/sink," Chinese Physics Letters, vol. 29, Article ID 054703, 2012.

[38] K. Sadeghy, H. Hajibeygi, and S.-M. Taghavi, "Stagnationpoint flow of upper-convected Maxwell fluids," International Journal of Non-linear Mechanics, vol. 41, no. 10, pp. 12421247, 2006. 